\title{
The Maximality of Cartesian Categories
}

\begin{abstract}
It is proved that equations between arrows assumed for cartesian categories are maximal in the sense that extending them with any new equation in the language of free cartesian categories collapses a cartesian category into a preorder. An analogous result holds for categories with binary products, which may lack a terminal object. The proof is based on a coherence result for cartesian categories, which is related to model-theoretical methods of normalization.
\end{abstract}

The equations between arrows assumed for cartesian categories are maximal in the sense that extending them with new equations collapses the categories into preorders (i.e. categories in which between any two objects there is at most one arrow). The equations envisaged for the extension are in the language of free cartesian categories generated by sets of objects, and variables for arrows don't occur in them. If such an equation doesn't hold in the free cartesian category generated by a set of objects, then any cartesian category in which this equation holds is a preorder. An analogous result is provable for categories with binary products, which differ from cartesian categories in not necessarily having a terminal object.

The proof of these results, which we are going to present below, is based on a coherence property of cartesian categories. This coherence, which is ultimately inspired by the geometric modelling of categories of [3], is related to model-theoretic methods of normalization. It permits to establish uniqueness of normal form for arrow terms without proceeding via the Church-Rosser property for reductions. It also yields an easy decision procedure for the commuting of diagrams in free cartesian categories.

\section{Cartesian categories}

A category with binary products is a category with a binary operation $\times$ on objects, projection arrows

$$
\begin{aligned}
& \mathbf{k}_{A, B}^{1}: A \times B \rightarrow A, \\
& \mathbf{k}_{A, B}^{2}: A \times B \rightarrow B,
\end{aligned}
$$

and the pairing operation that to a pair of arrows $(f: C \rightarrow A, g: C \rightarrow B)$ assigns the arrow $\langle f, g\rangle: C \rightarrow A \times B$. The arrows must satisfy the equations

$$
\begin{array}{ll}
(\beta 1) & \mathbf{k}_{A, B}^{1} \circ\langle f, g\rangle=f, \\
(\beta 2) & \mathbf{k}_{A, B}^{2} \circ\langle f, g\rangle=g, \\
(\text { distr }) & \langle f, g\rangle \circ h=\langle f \circ h, g \circ h\rangle, \\
(\eta) & \left\langle\mathbf{k}_{A, B}^{1}, \mathbf{k}_{A, B}^{2}\right\rangle=\mathbf{1}_{A \times B} .
\end{array}
$$

A category has a terminal object $\mathrm{T}$ iff it has the special arrows

$$
\mathbf{k}_{A}: A \rightarrow \mathrm{T},
$$


which satisfy the equation

(k) for $f: A \rightarrow \mathrm{T}, \quad f=\mathbf{k}_{A}$.

A cartesian category is a category with binary products and a terminal object.

In terms of the projection arrows and the pairing operation on arrows we may define in every category with binary products the following arrows:

$$
\begin{aligned}
\overrightarrow{\mathbf{b}}_{A, B, C}=\operatorname{def.} . & \left\langle\left\langle\mathbf{k}_{A, B \times C}^{1}, \mathbf{k}_{B, C}^{1} \circ \mathbf{k}_{A, B \times C}^{2}\right\rangle, \mathbf{k}_{B, C}^{2} \circ \mathbf{k}_{A, B \times C}^{2}\right\rangle \\
& \text { of type } A \times(B \times C) \rightarrow(A \times B) \times C, \\
\overleftarrow{\mathbf{b}}_{A, B, C}={ }_{\text {def. }} & \left\langle\mathbf{k}_{A, B}^{1} \circ \mathbf{k}_{A \times B, C}^{1},\left\langle\mathbf{k}_{A, B}^{2} \circ \mathbf{k}_{A \times B, C}^{1}, \mathbf{k}_{A \times B, C}^{2}\right\rangle\right\rangle \\
& \text { of type }(A \times B) \times C \rightarrow A \times(B \times C), \\
\mathbf{c}_{A, B}=\operatorname{def.} . & \left\langle\mathbf{k}_{A, B}^{2}, \mathbf{k}_{A, B}^{1}\right\rangle \\
& \text { of type } A \times B \rightarrow B \times A, \\
\mathbf{w}_{A}=\operatorname{def.} . & \left\langle\mathbf{1}_{A}, \mathbf{1}_{A}\right\rangle \\
& \text { of type } A \rightarrow A \times A .
\end{aligned}
$$

We may also define the product operation on arrows, which to a pair of arrows $(f: A \rightarrow B$, $g: C \rightarrow D$ ) assigns the arrow $f \times g: A \times C \rightarrow B \times D:$

$$
f \times g={ }_{\text {def. }}\left\langle f \circ \mathbf{k}_{A, C}^{1}, g \circ \mathbf{k}_{A, C}^{2}\right\rangle .
$$

In every cartesian category we also have the arrows

$$
\begin{aligned}
\boldsymbol{\sigma}_{A} \quad=\text { def. } & \left\langle\mathbf{k}_{A}, \mathbf{1}_{A}\right\rangle \\
& \text { of type } A \rightarrow \mathrm{T} \times A, \\
\boldsymbol{\delta}_{A} \quad=\text { def. } & \left\langle\mathbf{1}_{A}, \mathbf{k}_{A}\right\rangle \\
& \text { of type } A \rightarrow A \times \mathrm{T} .
\end{aligned}
$$

These definitions of category with binary products and cartesian category are equivalent to standard definitions (see [5], Part 0, Chapter 5, and Part I, Chapter 3), where

$$
A \stackrel{\mathbf{k}_{A, B}^{1}}{\longleftarrow} A \times B \stackrel{\mathbf{k}_{A, B}^{2}}{\longrightarrow} B
$$

is a product diagram with the desired universal property.

\section{Graphs of arrow terms in free cartesian categories}

Consider the free cartesian category Cart generated by a set of objects $\mathcal{O}$ called letters $(\mathrm{T}$ is not a letter). This category is the image of $\mathcal{O}$ under the left adjoint to the forgetful functor from the category of cartesian categories, with cartesian structure-preserving functors as arrows, to the category of sets (of objects). The construction of Cart out of syntactic material is explained in detail in [6] (Part I, Chapter 4; note that there the name "Cart" has a different meaning).

For an object $A$ of Cart let the letter length $|A|$ of $A$ be the number of occurrences of letters in $A$. For example, if $p$ and $q$ are letters, then $|((p \times q) \times p) \times(\mathrm{T} \times p)|$ is 4. Let $f: A \rightarrow B$ be an arrow term of Cart and let $|A|=n$ and $|B|=m$. To $f$ we associate a function $\Gamma_{f}$ from $\{1, \ldots, m\}$ to $\{1, \ldots, n\}$, called the graph of $f$. If $m=0$, then $\{1, \ldots, m\}$ is $\emptyset$. The function $\Gamma_{f}$ is defined by induction on the complexity of $f$ in the following manner.

If $f$ is of the form $\mathbf{1}_{A}: A \rightarrow A$, then $m=n$ and $\Gamma_{f}(i)=i$, where $i \in\{1, \ldots, m\}$. If $f$ is of the form $\mathbf{k}_{A, B}^{1}: A \times B \rightarrow A$, then $\Gamma_{f}(i)=i$, and if $f$ is of the form $\mathbf{k}_{A, B}^{2}: A \times B \rightarrow B$, then $\Gamma_{f}(i)=i+|A|$. If $f$ is of the form $\mathbf{k}_{A}: A \rightarrow \mathrm{T}$, then $\Gamma_{f}$ is the empty function (i.e., $\Gamma_{f}$ is $\emptyset: \emptyset \rightarrow\{1, \ldots,|A|\})$. 
If $f$ is of the form $\langle g, h\rangle: C \rightarrow A \times B$, with $g: C \rightarrow A$ and $h: C \rightarrow B$, then for $i \leq|A|$ we have $\Gamma_{f}(i)=\Gamma_{g}(i)$ and for $i>|A|$ we have $\Gamma_{f}(i)=\Gamma_{h}(i)$. Finally, if $f$ is of the form $h \circ g$, then $\Gamma_{f}(i)=\Gamma_{g}\left(\Gamma_{h}(i)\right)$.

For $f: A \rightarrow B$, the graph $\Gamma_{f}$ can be interpreted as connecting an occurrence of a letter $p$ in $A$ with a finite set of occurrences of $p$ in $B$ (this set may have more than one member, it may be a singleton, or it may be empty).

We can establish the following lemma.

Lemma 1 If $f=g$ in Cart, then $\Gamma_{f}=\Gamma_{g}$.

Proof: We proceed by induction on the length of the derivation of $f=g$ in Cart. In this induction it is essential to check that for the equations $(\beta 1),(\beta 2),($ distr $),(\eta)$ and $(\mathbf{k})$, the graphs of the two sides of the equation must be equal. The induction step, which involves the rules of symmetry and transitivity of equality, as well as congruence with composition and pairing, is quite trivial.

q.e.d.

We shall demonstrate the converse of this lemma in Lemma 3 below.

\section{Normal form of arrow terms in free cartesian categories}

From now on, identity of arrow terms of Cart will be taken up to associativity of composition. So, for example, $\mathbf{k}_{B, C}^{1} \circ\left(\mathbf{k}_{A, B \times C}^{2} \circ \mathbf{1}_{A \times(B \times C)}\right)$ will be considered to be the same arrow term as $\left(\mathbf{k}_{B, C}^{1} \circ \mathbf{k}_{A, B \times C}^{2}\right) \circ \mathbf{1}_{A \times(B \times C)}$, and we may omit parentheses in compositions. (Formally, we may work with equivalence classes of arrow terms.)

An arrow term of Cart is called an atomized $\mathbf{k}$-composition iff it is of the form $f_{n} \circ \cdots \circ f_{1}$ : $A \rightarrow B$, with $n \geq 1$, where $B$ is a letter and each $f_{i}$ is either of the form $\mathbf{k}_{C, D}^{1}$ or of the form $\mathbf{k}_{C, D}^{2}$.

Arrow terms of Cart in normal form are defined inductively as follows:

1. every arrow term $\mathbf{1}_{A}$ with $A$ a letter is in normal form;

2. every atomized $\mathbf{k}$-composition is in normal form;

3. every arrow term $\mathbf{k}_{A}$ is in normal form;

4. if $f: C \rightarrow A$ and $g: C \rightarrow B$ are in normal form, then $\langle f, g\rangle$ is in normal form.

The arrow terms defining $\overrightarrow{\mathbf{b}}, \overleftarrow{\mathbf{b}}, \mathbf{c}, \mathbf{w}, \boldsymbol{\sigma}$ and $\boldsymbol{\delta}$ arrows in section 1 are in normal form if $A, B$ and $C$ are letters.

For the normal form of arrow terms of Cart and their graphs we can prove the following fundamental lemma.

Lemma 2 Suppose $f, g: A \rightarrow B$ are arrow terms of Cart in normal form. Then $\Gamma_{f}=\Gamma_{g}$ iff $f$ and $g$ are the same arrow term.

Proof: Suppose $f, g: A \rightarrow B$ are different arrow terms of Cart in normal form. We shall show that in that case $\Gamma_{f} \neq \Gamma_{g}$ by induction on the number of pairing operations in $f$.

If this number is zero, then it must be zero too in $g$, because $B$ is a letter or T. If $f$ is of the form $\mathbf{1}_{A}$, then $g$ must also be $\mathbf{1}_{A}$, and if $f$ is of the form $\mathbf{k}_{A}$, then $g$ must also be $\mathbf{k}_{A}$. So $f$ cannot be $\mathbf{1}_{A}$ or $\mathbf{k}_{A}$. The only remaining possibility is that $f$ be an atomized $\mathbf{k}$-composition of the form $f_{n} \circ \cdots \circ f_{1}$. Then $g$ must be an atomized k-compsition, too; let it be of the form $g_{m} \circ \cdots \circ g_{1}$. It is excluded that $f$ be of the form

$$
f_{n} \circ \cdots \circ f_{n-k} \circ g_{m} \circ \cdots \circ g_{1}
$$


for $n-k \geq 1$, because the codomain of $g_{m}$ is a letter. Analogously, it is excluded that $g$ be of the form

$$
g_{m} \circ \cdots \circ g_{m-k} \circ f_{n} \circ \cdots \circ f_{1}
$$

for $m-k \geq 1$.

So for some $i$ we must have that $f_{i}$ and $g_{i}$ are different; let $j$ be the least such $i$. Then one of $f_{j}$ and $g_{j}$ is $\mathbf{k}_{C, D}^{1}$ while the other is $\mathbf{k}_{C, D}^{2}$, and the letter $B$ must occur in both $C$ and $D$. It follows easily that $\Gamma_{f} \neq \Gamma_{g}$.

If the number of pairing operations in $f$ is at least one, then $f$ is of the form $\left\langle f_{1}, f_{2}\right\rangle: A \rightarrow$ $B_{1} \times B_{2}$, and hence $g$, which is in normal form, must be of the form $\left\langle g_{1}, g_{2}\right\rangle$. Since $f$ is different from $g$, either $f_{1}$ and $g_{1}$ or $f_{2}$ and $g_{2}$ are two different arrow terms with identical domains and codomains, which are both in normal form. By the induction hypothesis, either $\Gamma_{f_{1}} \neq \Gamma_{g_{1}}$ or $\Gamma_{f_{2}} \neq \Gamma_{g_{2}}$, and in both cases we can infer $\Gamma_{f} \neq \Gamma_{g}$. This concludes the induction.

So, by contraposition, if $\Gamma_{f}=\Gamma_{g}$, then $f$ and $g$ are the same arrow term. Since the converse implication is trivial, this proves the lemma.

q.e.d.

Every arrow term of Cart can be reduced to normal form by using the following reductions:

\begin{tabular}{ccc} 
redexes & contracta \\
& identity reductions & \\
$f \circ \mathbf{1}_{A}$ & $f$ \\
$\mathbf{1}_{B} \circ f$ & $f$ \\
\multicolumn{2}{c}{ pairing reductions } \\
$\mathbf{k}_{A, B}^{1} \circ\langle f, g\rangle$ & $f$ \\
$\mathbf{k}_{A, B}^{2} \circ\langle f, g\rangle$ & $g$ \\
$\langle f, g\rangle \circ h$ & $\langle f \circ h, g \circ h\rangle$ \\
\multicolumn{2}{c}{ atomizing reductions } \\
$f: C \rightarrow A \times B \quad\left\langle\mathbf{k}_{A, B}^{1} \circ f, \mathbf{k}_{A, B}^{2} \circ f\right\rangle$ \\
$g: C \rightarrow \mathrm{T}$ & $\mathbf{k}_{C}$
\end{tabular}

We reduce an arrow term $t_{0}$ of Cart to normal form via a sequence $t_{0}, t_{1}, \ldots, t_{n}$, where $t_{i+1}$ is obtained from $t_{i}$ by a reduction step, which is a replacement of a subterm of $t_{i}$ that is a redex by the corresponding contractum. We call each $t_{i}, i<n$, in this sequence a candidate for reduction.

To obtain that every sequence of reduction steps terminates we must exclude in the first kind of atomizing reduction that the redex $f$ be of the form $\left\langle f_{1}, f_{2}\right\rangle$. We should exclude as well that it reduces to this form by other reductions. We must also ensure that the respective occurrence of $f$ does not belong to a subterm of the form $h \circ f$ where $h$ is either $\mathbf{k}_{A, B}^{1}$ or $\mathbf{k}_{A, B}^{2}$, or $h$ reduces to $\mathbf{k}_{A, B}^{1}$ or $\mathbf{k}_{A, B}^{2}$. In the second kind of atomizing reduction the redex $g$ should be different from $\mathbf{k}_{C}$. All this will be guaranteed if we add to the atomizing reductions the provisos we are going to formulate below. 
For an arrow term $t$ of Cart let $\gamma(t)$ be defined inductively as follows:

$$
\begin{aligned}
& \gamma\left(\mathbf{k}_{A}\right)=2, \\
& \gamma(t)=3 \text { if } t \text { is } \mathbf{1}_{A}, \mathbf{k}_{A, B}^{1} \text { or } \mathbf{k}_{A, B}^{2}, \\
& \gamma(g \circ f)=\gamma(g) \cdot \gamma(f), \\
& \gamma(\langle f, g\rangle)=\gamma(f)+\gamma(g)+1 .
\end{aligned}
$$

Let $\alpha(t)$ be $\gamma(t) \cdot n$, where $n$ is 1 if in $t$ there are pairing operations $\langle\ldots, \ldots\rangle$ within the scope of composition o; otherwise $n$ is 0 .

If $h_{2} \circ h_{1}$ is a subterm of an arrow term $t$, then $h_{1}$ and $h_{2}$ are called compositional subterms of $t$. Let a subterm $f$ of $t$ be called product-eliminative (for this terminology, suggested by natural deduction, see section 6 ) iff the pairing operation does not occur in $f$, and $f$ is not a compositional subterm of $t$.

The proviso for the first atomizing reduction says that the redex $f$ must be product-eliminative with respect to the candidate for reduction $t$, of which $f$ is a subterm, and $\alpha(t)=0$. The proviso for the second atomizing reduction says just that $g$ is not $\mathbf{k}_{C}$.

With these provisos, it is easy to check that $t$ is in normal form iff there are no redexes in it. (For that we rely on the fact that in Cart there is no arrow of type $\mathrm{T} \rightarrow A$ with $A$ a letter.)

For an object $A$ of Cart let the length of $A$ be the letter length $|A|$ of $A$ plus the number of occurrences of $\times$ and $\mathrm{T}$ in $A$ (i.e., the length of $A$ is the number of occurrences of symbols in $A$ ). For an arrow term $t$ of Cart let $\beta(t)$ be the sum of all the lengths of all the targets of producteliminative subterms of $t$. (If the same object is the target of $n$ product-eliminative subterms of $t$, then it is counted $n$ times in the sum $\beta(t)$.)

Let the degree of a candidate for reduction $t$ be the ordinal $\omega^{2} \cdot \alpha(t)+\omega \cdot \beta(t)+\gamma(t)$. It is easy to check that by replacing a redex of $t$ by a contractum the degree of the resulting arrow term strictly decreases. Then an induction up to $\omega^{3}$ (which is, of course, reducible to an ordinary induction up to $\omega$ ) shows that every sequence of reduction steps terminates.

Our provisos don't only yield that every sequence of reduction steps terminates in a normal form, but they optimize reductions in other respects, too. For our purposes, however, it is enough to know that some sequence of reduction steps terminates in a normal form, so that the provisos for atomizing reductions are not essential. But the provisos do help to make it clear that such a sequence exists. All the reductions above (without the provisos, and hence with the provisos, too) are covered by equations of Cart.

Lemmata 1 and 2 guarantee that if $f=g$ is satisfied in Cart and $f^{\prime}$ and $g^{\prime}$ are normal forms of $f$ and $g$, respectively, then $f^{\prime}$ and $g^{\prime}$ are the same arrow term. To prove that we conclude by Lemma 1 from $f=g, f=f^{\prime}$ and $g=g^{\prime}$, which we have in Cart, that $\Gamma_{f^{\prime}}=\Gamma_{g^{\prime}}$. Then by Lemma 2, it follows that $f^{\prime}$ and $g^{\prime}$ are the same arrow term.

So arrows of Cart have a unique normal form. Note that we have demonstrated this without appealing to the Church-Rosser property for our reductions.

\section{Coherence}

Before proving our theorem about the maximality of cartesian categories, we establish a lemma converse to Lemma 1.

Lemma 3 Suppose $f, g: A \rightarrow B$ are arrow terms of Cart. If $\Gamma_{f}=\Gamma_{g}$, then $f=g$ in Cart.

Proof: Suppose $f, g: A \rightarrow B$ are arrow terms of Cart such that $\Gamma_{f}=\Gamma_{g}$. Then let $f^{\prime}$ and $g^{\prime}$ be the normal forms of $f$ and $g$, respectively. Since $f=f^{\prime}$ and $g=g^{\prime}$ in Cart, by Lemma 1 we have $\Gamma_{f^{\prime}}=\Gamma_{g^{\prime}}$, and hence, by Lemma $2, f^{\prime}$ and $g^{\prime}$ are the same arrow term. Then by the symmetry and transitivity of equality it follows that $f=g$ in Cart. 
A notion of graph analogous to ours may be found in 14 (p. 94). A coherence result analogous to Lemma 3 is envisaged in [5] (p. 129), and is demonstrated in [7] (Theorem 2.2), 10 (Theorem 8.2.3, p. 207) and [8].

Lemmata 1 and 3 guarantee that there is a faithful cartesian functor from Cart to the category Finord $^{o p}$, whose objects are finite ordinals and whose arrows are arbitrary functions from finite ordinals to finite ordinals, with domains being targets and codomains sources. This functor is onto on objects and on arrows if Cart is generated by a nonempty set of letters. The product of Finord $^{o p}$, to which the product of Cart is mapped, is simply addition, and the terminal object is zero. If Cart is generated by a single object, then Finord $^{o p}$ is equivalent (but not isomorphic) to Cart: it is the skeleton of Cart.

Our demonstration of uniqueness of the normal form in the preceding section, which did not appeal to the Church-Rosser property of reductions, is akin to model-theoretical methods of normalization (see [1] and references therein). In the spirit of these methods, a computation without reductions of the normal form of an arrow term $f$ of Cart consists in finding $\Gamma_{f}$, and then constructing out of $\Gamma_{f}$ an arrow term of Cart in normal form whose graph is $\Gamma_{f}$.

The equivalence between $f=g$ and $\Gamma_{f}=\Gamma_{g}$, which follows from Lemmata 1 and 3, provides an easy decision procedure for commuting of diagrams in Cart. To check whether $f=g$ in Cart, we just have to check whether $\Gamma_{f}=\Gamma_{g}$. (An alternative decision procedure for the commuting of diagrams in Cart can be obtained via reduction to normal form, according to section 3.)

\section{The maximality theorem}

For arrow terms $f, g: A \rightarrow B$ of Cart, we say that $f=g$ holds in a cartesian category $\mathcal{C}$ iff for every cartesian functor $F$ from Cart to $\mathcal{C}$ we have $F(f)=F(g)$ in $\mathcal{C}$.

Then we can prove our theorem.

Theorem Suppose $f, g: A \rightarrow B$ are arrow terms of Cart such that in Cart we don't have $f=g$. If $f=g$ holds in a cartesian category $\mathcal{C}$, then $\mathcal{C}$ is a preorder.

Proof: Let $f, g: A \rightarrow B$ be arrow terms of Cart such that in Cart we don't have $f=g$. By Lemma 3 , it follows that $\Gamma_{f} \neq \Gamma_{g}$. So there must be an occurrence of a letter $p$ in $B$ such that if $p$ is the $i$-th letter symbol of $B$, counting from the left, then $\Gamma_{f}(i) \neq \Gamma_{g}(i)$. Consider the substitution instances $f^{\prime}, g^{\prime}: A^{\prime} \rightarrow B^{\prime}$ of $f$ and $g$ obtained by replacing every letter by $p$. So the only letter in $A^{\prime}$ and $B^{\prime}$ is $p$. It is clear that $\Gamma_{f^{\prime}}(i) \neq \Gamma_{g^{\prime}}(i)$.

Then there is an arrow term $h: p \times p \rightarrow A^{\prime}$ of Cart made of possibly multiple occurrences of the arrow terms $\mathbf{1}, \overrightarrow{\mathbf{b}}, \overleftarrow{\mathbf{b}}, \mathbf{c}, \mathbf{w}, \boldsymbol{\sigma}$ and $\boldsymbol{\delta}$, together with the product operation on arrows and composition, such that $\Gamma_{h}\left(\Gamma_{f^{\prime}}(i)\right)=1$ and $\Gamma_{h}\left(\Gamma_{g^{\prime}}(i)\right)=2$.

There is also an arrow term $j: B^{\prime} \rightarrow p$ that is either $\mathbf{1}_{p}$ or an atomized $\mathbf{k}$-composition (see section 3) such that $\Gamma_{j}(1)=i$. Then $j \circ f^{\prime} \circ h$ and $j \circ g^{\prime} \circ h$ are both of type $p \times p \rightarrow p$, while $\Gamma_{j \circ f^{\prime} \circ h}=\Gamma_{\mathbf{k}_{p, p}^{1}}$ and $\Gamma_{j \circ g^{\prime} \circ h}=\Gamma_{\mathbf{k}_{p, p}^{2}}$. Therefore, by Lemma 3, in Cart we have $j \circ f^{\prime} \circ h=\mathbf{k}_{p, p}^{1}$ and $j \circ g^{\prime} \circ h=\mathbf{k}_{p, p}^{2}$. So these two equations hold in every cartesian category $\mathcal{C}$. If $f=g$ holds in $\mathcal{C}$, then $f^{\prime}=g^{\prime}$ holds too, and so $\mathbf{k}_{p, p}^{1}=\mathbf{k}_{p, p}^{2}$ holds in $\mathcal{C}$.

Now suppose $s, t: C \rightarrow D$ are two arrows of $\mathcal{C}$. Then in $\mathcal{C}$ we have

$$
\mathbf{k}_{D, D}^{1} \circ\langle s, t\rangle=\mathbf{k}_{D, D}^{2} \circ\langle s, t\rangle,
$$

and so $s=t$, by $(\beta 1)$ and $(\beta 2)$.

q.e.d.

An analogous maximality theorem can be proved for categories with binary products. For that we have to replace Cart by the free category with binary products Cart $^{-}$generated by a set of 
letters. Graphs for the arrow terms of $\mathbf{C a r t}^{-}$are defined by just omitting the clause for $\Gamma_{\mathbf{k}_{A}}$ in section 2. In the definition of the normal form in section 3 we omit clause (3), and the second kind of atomizing reduction in the same section is now superfluous. Exact analogues of Lemmata 1, 2 and 3 are provable as before, while in the proof of the Theorem we need not mention $\sigma$ and $\boldsymbol{\delta}$ arrow terms in the second paragraph. This way we show that, if $f, g: A \rightarrow B$ are arrow terms of $\mathbf{C a r t}^{-}$such that in $\mathbf{C a r t}^{-}$we don't have $f=g$, and $f=g$ holds in a category with binary products $\mathcal{C}$, then $\mathcal{C}$ is a preorder.

\section{A logical conclusion}

It is well known that equations between arrows in cartesian categories correspond to equivalence between deductions in conjunctive logic, including the constant true proposition. This equivalence induces an equality relation on equivalence classes. As far as $\times$ is concerned, this equality between deductions permits us to reduce every deduction into one in atomized normal form, where elimination rules, which correspond to $\mathbf{k}^{1}$ and $\mathbf{k}^{2}$ arrows, precede introduction rules, which correspond to the pairing operation, and the middle part between eliminations and introductions is atomic. This atomized normal form corresponds to the normal form of arrow terms of Cart in section 3 .

The import of the maximality of cartesian categories for logic is that the usual assumptions for equivalence between deductions in conjunctive logic are optimal. These assumptions are wanted, because they are induced by normalization of deductions, and no assumption is missing, because any further equation would equate all deductions that share premises and conclusions.

Of course, by duality, this solves the problem for disjunctive logic, including the constant absurd proposition or not. This logic corresponds to categories with binary coproducts, which when they have an initial object are "cocartesian" categories, i.e. cartesian categories with arrows reversed.

It is natural to enquire whether similar maximality results hold for other sorts of categories of interest to logic. An exactly analogous result for cartesian closed categories is proved in [9] (Theorem 1) and [2]. However, this result is independent from the maximality result for cartesian categories proved in this note: neither result can be inferred from the other.

\section{References}

[1] D. Čubrić, P. Dybjer and P. J. Scott, Normalization and the Yoneda embedding, Mathematical Structures in Computer Science vol. 8(1998), 153-192.

[2] K. Došen and Z. Petrić, The maximality of the typed lambda calculus and of cartesian closed categories, preprint IRIT 98R, 1998.

[3] S. Eilenberg and G. M. Kelly, A generalization of the functorial calculus, Journal of Algebra vol. 3(1966), 366-375.

[4] G. M. Kelly, Many-variable functorial calculus I, in: S. Mac Lane ed., Coherence in Categories, Lecture Notes in Mathematics 281, Springer, Berlin, 1972, pp. 66-105.

[5] G. M. Kelly, An abstract approach to coherence, in: S. Mac Lane ed., Coherence in Categories, Lecture Notes in Mathematics 281, Springer, Berlin, 1972, pp. 106-147.

[6] J. Lambek and P. J. Scott, Introduction to Higher-Order Categorical Logic, Cambridge University Press, Cambridge, 1986.

[7] G. E. Mints, Category theory and proof theory (in Russian), in: Aktual'nye voprosy logiki i metodologii nauki, Naukova Dumka, Kiev, 1980, pp. 252-278 (English translation, with permuted title, in: G. E. Mints, Selected Papers in Proof Theory, Bibliopolis, Naples, 1992). 
[8] Z. Petrić, Coherence in substructural categories (to appear).

[9] A. K. Simpson, Categorical completeness results for the simply-typed lambda-calculus, in: M. Dezani-Ciancaglini and G. Plotkin eds, Typed Lambda Calculi and Applications (Edinburgh, 1995), Lecture Notes in Computer Science 902, Springer, Berlin, 1995, pp. 414-427.

[10] A. S. Troelstra and H. Schwichtenberg, Basic Proof Theory, Cambridge University Press, Cambridge, 1996. 